\title{
A Novel Effective Treatment of Advanced Prostate Cancer: A Single Case Report
}

\section{Rolf Seljelid ${ }^{1 *}$ | Jarl Bøgwald ${ }^{2}$}

*Correspondence: Rolf Seljelid

Address: ${ }^{1}$ Faculty of Medicine, UiT The Arctic University of Norway, Tromsoe, Norway; ${ }^{2}$ Faculty of Biosciences, Fisheries and Economics, UiT The Arctic University of Norway, Tromsoe, Norway

e-mail $₫$ rseljelid@gmail.com

Received: 08 November 2021; Accepted: 17 November 2021

Copyright: (C) 2021 Seljelid R. This is an open-access article distributed under the terms of the Creative Commons Attribution License, which permits unrestricted use, distribution, and reproduction in any medium, provided that the original work is properly cited.

\section{ABSTRACT}

A novel treatment for prostate cancer, based on the $\mathrm{T}_{\mathrm{vec}}$ /pembroluzimab technology - supported by activation of innate immunity is reported. The effect has been striking. Reduction of PSA from $60 \mu \mathrm{g} /$ Liter to undetectable in 6 weeks, reduction of serum LDH from almost $1000 \mathrm{U} / \mathrm{L}$ to under 200 (normal) in 8 weeks, extensive necrosis in the tumor tissue as revealed by MR. The treatment gave no serious side-effects.

Keywords: Prostate, Cancer, $T_{\text {vec, }}$ Pembroluzimab, Innate Immunity

\section{Introduction}

In October 2017 one of us (RS) was diagnosed with cancer of the prostate. We immediately looked for possibilities of treatment. There are few effective treatments for advanced cancer of the prostate: Radical surgery is excluded when the cancer has penetrated outside the gland - which is often the case; irradiation and chemotherapy often of limited use because of serious side effects; immunotherapy can in most cases not be applied because of scarcity of cancer antigens in the tumor (Li et al., 2010).

We will here report one case of a successful treatment based on modified immunisation $\left(\mathrm{T}_{\text {vec }}\right.$ and anti PD1, pembrolizumab), combined with activation of innate immunity with $\beta 1-3$ glucan. The treatment was without serious side-effects, resulted in rapid and long-lasting normalization of prostate specific antigen (PSA) and lactate dehydrogenase (LDH) in blood serum (Fig. 1AB) - as well as extensive necrosis of tumor tissue as revealed by MR examination (Fig. 1CD).

\section{Case Report}

The patient is 87 years old, generally in good health, active. He fell and had a compression fracture 
of columna Th 11 in 2010, same year bypass surgery with good result, coxarthrosis bilaterally, prosthesis surgery left side 2005. Diagnosed with cancer of prostate in autumn 2017, penetration of capsule towards vesicula seminalis, possible small metastasis os ileum right hand side, and possible small metastasis in columna L2 as revealed by magnetic resonance (MR) examination. The histological examination showed: mostly Gleason 5-7, in one spot Gleason 9. PSA 10. Received antihormon treatment (Goserelin) from May 2018 till present, radical x-ray treatment July- August 2018. Zoledronic acid every third months from April 2018 to present, Xofigo (Radium ${ }^{223}$ Ra dichloride) March to April 2020. MR May 2020: Progression of metastasis in os ileum with extensive soft tissue component, also progression in L2, new growth in L5 and right hand femur diafysis. In October 2020 palliative x-ray 8 Gy $\mathrm{x} 1$ at os ileum and L2. Two small inguinal lymph nodes (approx. 2 x $1 \mathrm{~cm}$ ) were noticed. The patient had moderate discomfort right hand iliac region from time of diagnosis. None of these treatments had a lasting effect.

We decided based on available literature (Kohlhapp and Kaufman, 2016; Trehub and Gavrilov, 2021; Sweeney and Halldén, 2012) to try $\mathrm{T}_{\mathrm{vec}}$ treatment and PD1 inhibition followed by activation of innate immunity with SBG gel glucan (ß1-3 glucan) (Seljelid et al., 1981).

\section{Clinical Experiment}

$\mathrm{T}_{\text {vec }}$ treatment was started on October 21.2020 when $10^{6} \mathrm{PFU}$ was injected into the two left hand side inguinal lymph nodes. $200 \mathrm{mg}$ Pembrolizumab was given intravenously. Five mg ß1-3 glucan per os was administered later the same day. On the following dates, November 11, December 2, and December 17, $10^{8} \mathrm{PFU} \mathrm{T}_{\text {vec }}$ were injected into the inguinal lymph nodes and $200 \mathrm{mg}$ Pembrolizumab was administered intravenously. $5 \mathrm{mg}$ ß1-3 glucan was given per os later each day. No side-effects were noticed, except for a slight flu-like condition a few hours following the $\mathrm{T}_{\mathrm{vec}}$ injection. After the second round of parenteral medication a slight reduction in serum thyroid hormone developed $(-10 \%$ from normal). Except for these two observations, no significant side-effects were noticed.

Pronounced reduction of PSA was recorded, from $60 \mu \mathrm{g} /$ Liter at the start of treatment on October 21 to nondetectable on December 17 and onward to present (Fig. 1A). During the same period LDH was reduced from 915 to $173 \mathrm{U} / \mathrm{L}$ (normal) (Fig. 1B). MR on the 7th October (before treatment) showed progressive vital growth of tumor tissue in all noted locations (Fig. 1C). MR on January 5 (after treatment) showed extensive necrosis of tumor tissue in all locations (os ileum, columna and elsewhere, also in soft tissues (Fig. 1D). 

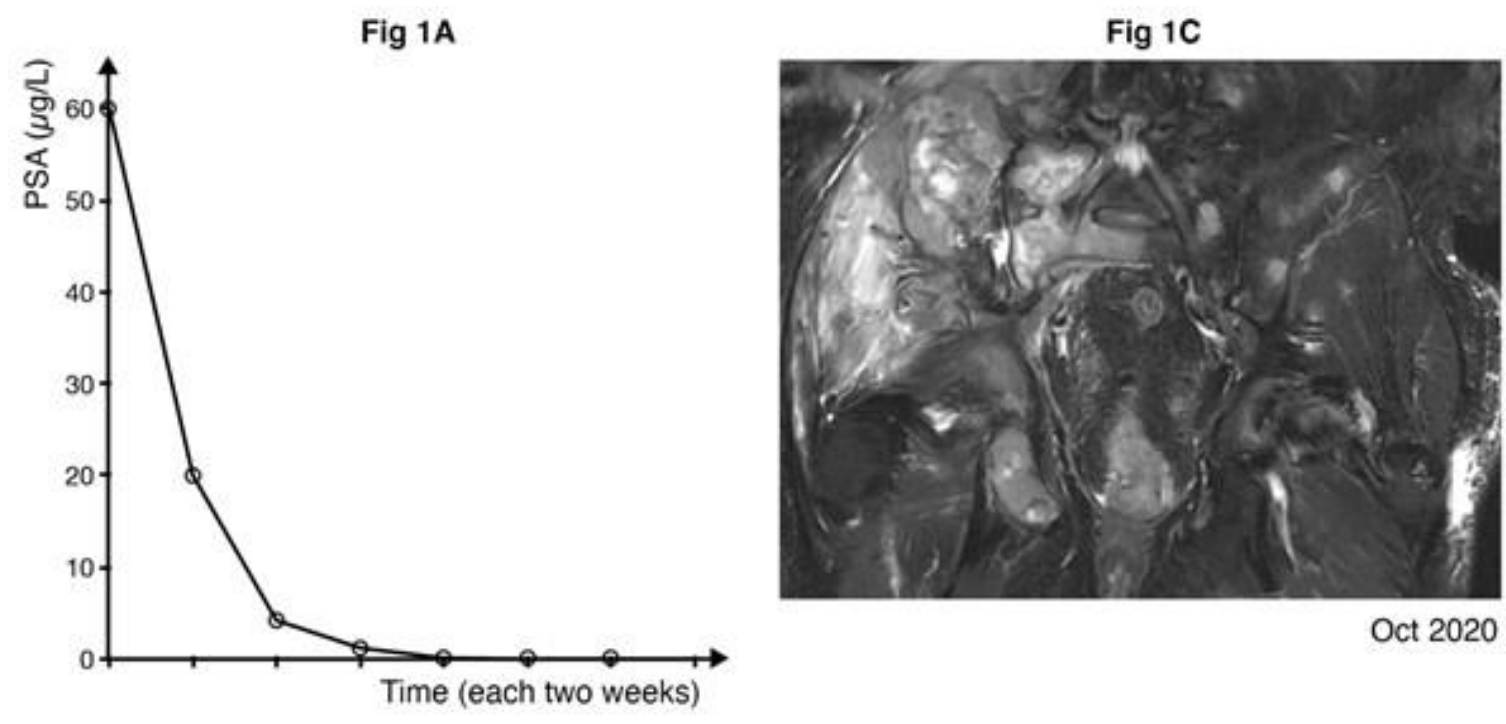

\section{Fig $1 \mathrm{C}$}

Oct 2020

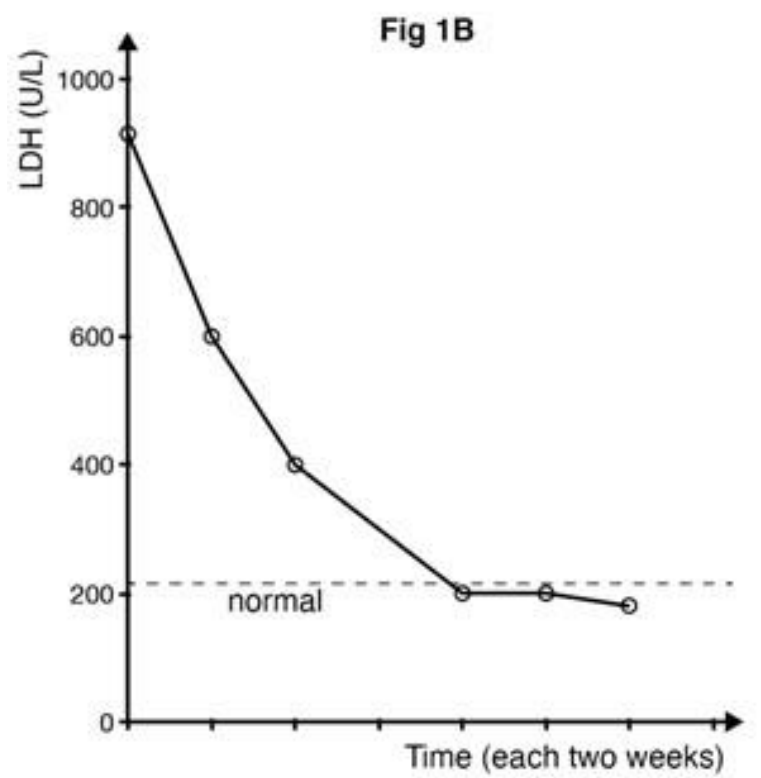

Fig 1D

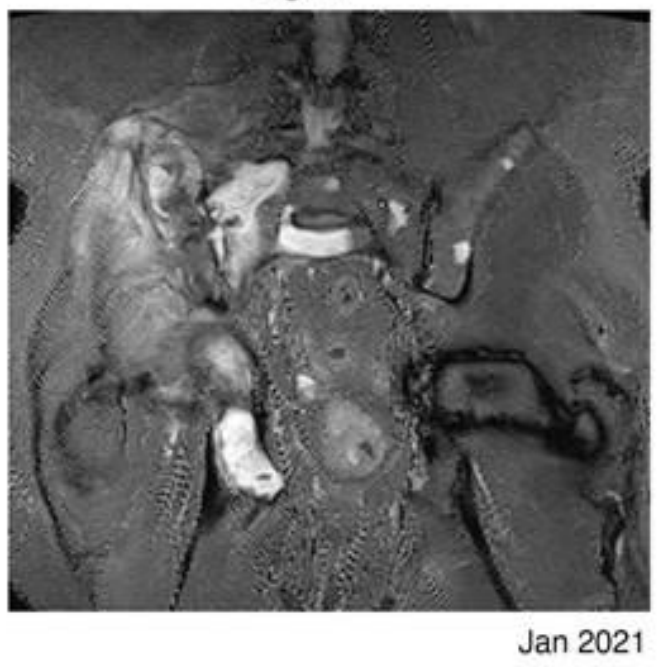

Figure 1: (1A) PSA reduced from $60 \mu \mathrm{g} / \mathrm{L}$ to zero in 6 weeks. (1B) LDH, reduced from 915U/L to 173 (normal) in 8 weeks. (1C) MR picture in right os ileum October 2020, before treatment: “vital tumor tissue, small bone fractures” (Dr Wolfgang Picker). (1D) Picture in right os ileum January 2021, two and a half months after beginning of treatment: "extensive necrosis of tumor tissue, fragments of bone" (Dr Wolfgang Picker).

\section{Discussion}

All vertebrates including humans have two immune systems, the innate and the adaptive, sometimes functionally overlapping, but more often with distinctive features and functions. The adaptive system, often referred to as 'the immune system' is organized in clones, which confer extreme specificity, relative slow response in primary reactions and limited destructive capacity. The innate system, based on the function of a large number of almost identical cells, the macrophages, has limited 
specificity, very fast response and extreme destructive capacity - especially when preactivated. The adaptive system -found in all vertebrate species - is based on the function of lymphocytes, T and B cells. The innate system in higher vertebrates including humans, is mainly based on the function of monocytes/macrophages, found in all vertebrates, and with minor modifications also in invertebrates (Paul, 2013; Abbas et al., 2017; Gordon, 2007).

1. Activation of innate immunity. Many years ago, we looked for, and found polysaccharides that stimulated macrophages (Seljelid et al., 1981). Mouse macrophages in vitro were treated with 42 different polysaccharides (glucans and glycans) water soluble, gels or insoluble. Activation of macrophages (innate immunity) was evaluated by cell size, $5^{\prime}$ nucleotidase activity and incorporation of C14 glucosamine (glycoprotein synthesis).

All activating polysaccharides were insoluble or gels, but not all insolubles were activating (ex chitin, cellulose). Polysaccharides with ß1-3 bonding were all stimulatory, the strongest stimulating glucans were exclusively ß1-3 bonded. None of the watersoluble glycans were stimulatory. (Seljelid et al., 1981) A watersoluble, stimulatory ß1-3 glucan suitable for in vivo experiments, aminated ß1-3 glucan, was produced by treatment of native, insoluble ß1-3 glucan with chloroacetylaldehyde dimethyl acetal followed by reductive amination. (Bogwald et al., 1986) We also made conjugates of $0.5 \mathrm{~mm}$ plastic beads with amino groups courtesy professor John Ugelstad, NTNU, Trondheim, Norway.

2. Pretreatment with aminated $ß 1-3$ glucan in mice with lethal bacterial infection. Pretreatment with aminated ß1-3glucan conferred strong protection against E.coli and pneumococci. (3 $\mu$ g aminated ß1-3glucan injected intravenously 24 hours before infection produced total survival, whereas all nonmedicated mice died within 12 hours). When glucan plastic bead conjugates were given as intraperitoneal pretreatment, the effect was even stronger. $(1.5 \mu \mathrm{g}$ ß1-3glucan attached to plastic beads gave maximum protection) (Seljelid et al., 1987).

3. Effect of treatment with ß1-3 glucan in mice with implanted tumors. Meth A sarcoma was inoculated in the skin of hybrid CB6 F1 (Balb/c x C 57131/6) and grew progressively. There was a characteristic period of slow growth around days 8-14, supposedly caused by a weak immune reaction after which the initial rapid growth resumed (Seljelid, 1986). When $5 \mathrm{mg}$ of pure water-soluble aminated ß1-3 glucan was injected intravenously or intraperitoneally on day 7, there was an almost immediate visible effect (Seljelid, 1986). At 1 hour after the injections the tumor in mice treated with saline intravenously were reddish and bulging, the tumors in mice having received ß1-3 glucan intravenously or intraperitoneally appeared collapsed and whitish-grey. At 6 hours this was even more pronounced. Histological examination showed acute inflammation around the tumor in the glucan- 
treated animals with thickening of the walls of small vessels, stasis of red blood cells and perivascular collection of mononuclear cells. At day 21 after treatment, tumor tissues were completely replaced by scar tissue. (Seljelid, 1989). This observation was also done after 200 days. The animals at this time point appeared to be unaffected and vital. In TxB animals, (thymectomized, $x$-ray irradiated and bone marrow transplanted) there was no effect of ß1-3 glucan at days 8-14. To decide whether the ß13glucan regression was peculiar to the Meth A tumor, we also performed experiments with sarcoma SA1, syngeneic in A strain mice. The results were essentially identical to those documented with the Meth A, the only difference being that in about one third of the cases, tumors reappeared and resumed growth after 18-21 days.

In tumor immunotherapy it would seem advantageous to be able to combine the specificity of the adaptive system, with the destructive capacity of an activated innate system. This is the underlying idea of the clinical experiment reported here. We claim that in this one patient with advanced cancer of the prostate it has been possible to obtain exactly that: the combined effect of oncolytic virus, plus inhibition of the PB1 blocking mechanisms, and added necessary destructive capacity by systemic administration of ß1-3 glucan gave apparent total regression of the malignant tissue. If the effect can be reproduced in a number of patients, it will represent a significant step forward in the management of prostate cancer, possibly also in other malignancies.

Comprehensive studies have laid down in great detail the molecular interactions following $\mathrm{T}_{\text {vec }}$ treatment (Kohlhapp and Kaufman, 2016; Trehub and Gavrilov, 2021) implying an explanation of the anti-cancer effect. However, the actual clinical results have not been equivalent. In large stage 2, stage 3 studies an average of a few weeks extended life expectancy is all that have been achieved (Sweeney and Halldén, 2012). This must mean that one or more essential factors have gone missing in the analysis. The involvement of activated innate immune mechanisms would be an obvious candidate.

\section{Conclusion}

One cannot draw general conclusions from single case observations like the one reported here. That will have to await clinical studies with a large number of patients, suitable for statistical analysis. But if one includes results from animal experiments - like ours from around 1990 (Seljelid, 1986; Seljelid et al., 1987; Seljelid, 1989), repeated twenty times or more with the same results - in a general biological consideration, then it appears to be a tenable position to hold that addition of activated innate immune cells will significantly increase the efficiency of certain immune reactions, like e. g. in immunotherapy of malignant tumors. 


\section{References}

Abbas AK, Lichtman AH, Pillai S. Cellular and Molecular Immunology. 9th edition, Elsevier, 2017

Bogwald J, Hoffman J, Seljelid R. Coupling of polysaccharides by means of chloroacetaldehyde dimethyl acetal to amines or protein by reductive amination. Carbohydrate Res 1986; 148: 101-107.

Gordon S. The macrophage: Past, present and future. Eur J Immunol 2007; 37: S9-S17.

Kohlhapp FJ and Kaufman HL. Molecular Pathways: Mechanism of Action for Talimogens Laherparepvcc, a New Oncolytic Virus Immunotherapy. Clinical Cancer Res 2016; 22: 1048.

Li Y, Cozzi PJ, Russell P. Promising tumor-associated antigens for future prostate cancer therapy. Medicinal Research Reviews 2010; 30: 67-101.

Paul WE. Fundamental Immunology. Wolters Kluwer Health/Lippincott Williams \& Wilkins, Philadelphia, 2013.

Seljelid R, Bogwald J, Lundwall A. Glycan stimulation of macrophages in vitro. Exp Cell Res 1981; 13: 121-129.

Seljelid R, Rasmussen LT, Larm 0, Hoffman J. The protective effect of beta 1-3 D-glucan derivatized plastic beads against Escherichia coli infection in mice. Scand J Immunol 1987; 25: 55-60.

Seljelid R. A watersoluble aminated ß1-3 glucan Derivative causes Regression of Solid Tumors in mice. Bioscience Reports 1986; 9: 845-851.

Seljelid R. Tumour Regression after Treatment with aminated ß1-3 polyglucose is initiated by circulatory failure. Scand J Immunol 1989; 29: 181-192.

Sweeney K and Halldén G. Oncolytic adenovirus - mediated therapy for Prostate cancer. Oncolytic Virotherapy 2012; 5: 45-57.

Trehub Y and Gavrilov A. Oncolytic virus in Immunotherapeutic Agents. In Springer Nature Cancer Immunology Rezaei N. (Ed.) 2021; 2021: 509-541. 